\title{
Estado actual de la biotecnología en Costa Rica
}

\author{
Marta Valdez ${ }^{1,2}$, Rebeca López ${ }^{3}$ \& Luis Jiménez ${ }^{2,3}$ \\ 1 Escuela de Biología, Universidad de Costa Rica, San José, Costa Rica; mvaldez@biologia.ucr.ac.cr. \\ 2 Vicerrectoría de Investigación, Universidad de Costa Rica, San José, Costa Rica. \\ 3 Centro Nacional de Ciencia y Tecnología de Alimentos, Universidad de Costa Rica, San José, Costa Rica.
}

Recibido 06-II-2004. C Corregido 24-VIII-2004. Aceptado 24-VIII-2004.

\begin{abstract}
Current estate of biotechnology in Costa Rica. A study was carried out on the construction of indicators in biotechnology in Costa Rica as part of the project "SYMBIOSIS, Cooperative Program for the Construction of Indicators in Biotechnology adapted to Latin American and Caribbean countries, to motivate the application and transference of industrial technologies". The study focused on two units: researchers and research projects developed in Costa Rica, between 1998 and 2002. For researchers, information was collected about indicators related to sex, age, teaching activities, number of projects, academic degree, area of speciality and number of publications. For research projects we obtained information about: speciality, sector of application, duration of projects and number of researchers per project. Very interesting results include the high participation of the women in this area of investigation (54\%); the low participation of young researchers $(13 \%$ younger than 30), and a high proportion of the investigators that are responsible for 4 or more projects $(42 \%)$. With relation to the specialities of the projects, the majority are in the category Bio-Agro (39\%) whereas in Acuaculture only $1 \%$ was found. The sectors of application with the most number of projects are: Agriculture and Livestock (37\%) and Human Health (35\%). The main strengthts and limitatations for the development of biotechnology in Costa Rica are discussed. Rev. Biol. Trop. 52(3): 733-743. Epub 2004 Dic 15.
\end{abstract}

Key words: Costa Rica, Biotechnology, Agricultural biotechnology, Human health, Indicators.

Palabras clave: Costa Rica, Biotecnología, Indicadores, Biotecnología agrícola, Salud humana.

El término "biotecnología" empezó a usarse alrededor del año 1960 para designar todas las técnicas cuyo instrumento de trabajo son los seres vivos y sus aplicaciones. No obstante, éstas son muy antiguas, se inician con el establecimiento de la civilización humana. Hace más de 6000 años, por ejemplo, los sumerianos y babilonios conocían ya la preparación de varios tipos de cervezas a partir de macerados y extractos de granos (Sasson 1988, 1991). El desarrollo histórico de la biotecnología muestra la gran cantidad de aplicaciones biotecnológicas para la producción de bienes y servicios a lo largo de la historia del desarrollo de las civilizaciones humanas (Sasson 1985).

Actualmente, la biotecnología comprende una amplia variedad de conocimientos y tecnologías que incluyen disciplinas básicas y aplicadas, tales como la genética, biología celular y molecular, química, bioprocesos, biofermentación, aplicaciones farmacéuticas, aplicaciones médicas, y recientemente, la genómica, bioinformática, ingeniería genética y la proteómica.

A nivel mundial, la biotecnología ha tenido un impacto positivo en el área de la salud, especialmente en las ciencias médicas y farmacéuticas. Se ha dado un avance excepcional en los métodos de diagnóstico y tratamiento de enfermedades y deficiencias genéticas. De manera similar, la biotecnología vegetal ha tenido profunda repercusión en las prácticas agrícolas y en el mejoramiento de la calidad y cantidad de alimentos y de plantas cultivables de interés 
comercial (Serageldin 1999). En consecuencia, su aplicación a la resolución de problemas que afectan el desarrollo y productividad de las economías de países del Tercer Mundo es impostergable. La biotecnología puede tener una incidencia importante en los países en desarrollo en las siguientes áreas: i) agricultura, horticultura y forestería; ii) alimentos y nutrición; iii) producción y salud animal y vegetal; iv) medicina y salud pública; v) productos farmacéuticos; vi) producción de energía; vii) conversión de desechos y subproductos agrícolas (Zeledón 1988).

En Costa Rica, especialmente en la Universidad de Costa Rica (UCR), se ha logrado un desarrollo notable en el campo de la biotecnología en los últimos años, sobre todo en los sectores agrícola y de salud pública, tanto a nivel académico como empresarial (CONARE 2003). Sin embargo, existe una gran dispersión de esfuerzos y recursos que es indispensable articular. Es entonces de fundamental importancia, realizar actividades tendientes a la integración interdisciplinaria de unidades y grupos de investigación que realizan trabajos en el campo de la biotecnología, con el fin de potenciar su contribución al desarrollo del país. Una primera etapa debe consistir en un adecuado conocimiento de los grupos de investigación y de las actividades biotecnológicas que se realizan en los diversos países, con miras al fortalecimiento de las alianzas estratégicas para el uso de los recursos disponibles, de manera eficiente. En ese sentido, el proyecto SIMBIOSIS, en su "Programa Cooperativo para la Construcción de Indicadores en Biotecnología y Tecnología de Alimentos, adaptados a los países de América Latina y el Caribe, para motivar la aplicación y transferencia de tecnologías industriales", ha sido una excelente iniciativa. Este proyecto es auspiciado por la Organización de Estados Americanos, para la integración de dicho conocimiento, tanto en Costa Rica como en los otros países participantes: México, Colombia y Venezuela (Testa 2003).

El objetivo del presente trabajo fue analizar el estado actual de la biotecnología en Costa Rica, en el periodo de 1998 a 2002, dentro del marco del proyecto SIMBIOSIS, y así, generar el conocimiento necesario para poder definir estrategias de investigación y desarrollo en el país.

\section{MATERIALES Y MÉTODOS}

Para recopilar la información, se diseñó un formulario para cada una de las unidades de investigación de Costa Rica, dedicadas a actividades biotecnológicas, donde debían completarse los datos referentes a proyectos e investigadores. Se elaboraron las respectivas listas de las unidades de estudio con base en: i) búsqueda de investigadores y proyectos, por medio de descriptores biotecnológicos, en las bases de datos disponibles; ii) revisiones de guías telefónicas; iii) estudios previos realizados en el país en este tema (UCR 2001); iv) información disponible en internet. Cuando la información en las bases de datos se encontraba desactualizada o no estaba disponible, se consultó directamente en los centros de investigación y con los investigadores.

El estudio se enfocó en dos ítemes: investigadores y proyectos de investigación desarrollados en el período comprendido entre 1998 y 2002. Los formularios fueron distribuidos por distintas vías según la conveniencia: por medio de fax, correo electrónico, correo postal o entrega personal. Los indicadores fueron estimados utilizando los parámetros establecidos en el proyecto "SIMBIOSIS: Programa Cooperativo para la Construcción de Indicadores en Biotecnología y Tecnología de Alimentos, adaptados a los países de América Latina y el Caribe, para motivar la aplicación y transferencia de tecnologías industriales", auspiciado por la OEA.

Para el ítem "investigadores", se solicitó información sobre ocho indicadores, relacionados con aspectos como género, edad, desempeño como docentes, número de proyectos, funciones, grado académico, área de especialidad y publicaciones. Los indicadores sobre los proyectos de investigación se vinculan con: especialidades, sector socio-económico de 
aplicación, duración y número de investigadores por proyecto. Para identificar las especialidades y sectores de aplicación de los proyectos, se utilizó como punto de partida la clasificación propuesta por Ver van Beuzekon (2001) para ser utilizada por los países miembros de la Organización Económica de Cooperación y Desarrollo (OECD). Esta clasificación incluye desde actividades de ciencia básica, hasta la identificación de resultados y de aplicaciones industriales. En la presente investigación se recolectó en total, la información de 17 centros de investigación del sector público y de tres del sector privado. Con la información recolectada se construyó una base de datos en el programa Access de Microsoft ${ }^{\mathrm{TM}}$, a partir de la cual se obtuvieron los indicadores.

\section{RESULTADOS}

Se obtuvo la información de 95 investigadores principales de los proyectos. No se incluyeron estudiantes tesiarios, ni asistentes de investigación. Se conoce que faltó información de algunos investigadores de las siguientes instituciones: Centro Agronómico Tropical de Investigación y Enseñanza (CATIE), Universidad Nacional (UNA) e Instituto Tecnológico de Costa Rica (ITCR), que no respondieron a la solicitud de información.

Hay una alta participación femenina (54\%) en la investigación biotecnológica del país (Fig. 1). La figura 2 muestra que la mayoría son nacionales $(87 \%)$. En relación con la edad, puede observarse la baja participación de recursos humanos jóvenes (Fig. 3). Sólo un $13 \%$ de ellos tiene menos de 30 años, un $64 \%$ tienen edades comprendidas entre los 30 y los 50 años, y un $18 \%$ tiene más de 50 años.

Una alta proporción de investigadores (42\%), tuvo más de cuatro proyectos de investigación bajo su responsabilidad, en el período del presente estudio (Fig. 4). Por otro lado, el $40 \%$ de los investigadores tienen el grado académico de Maestría, y un 35\% el de Doctorado

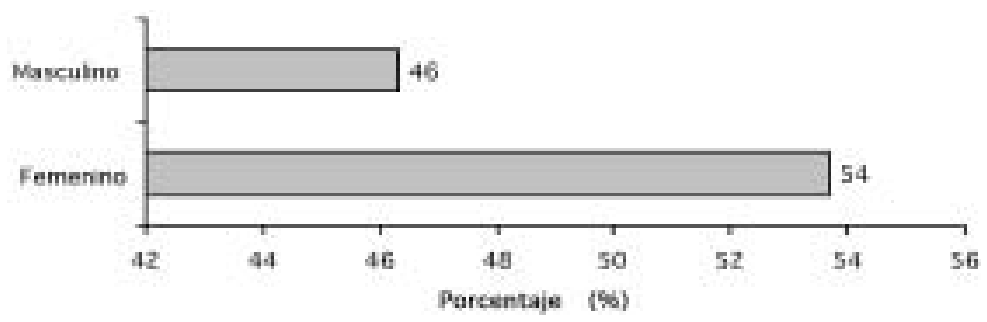

Fig. 1. Porcentaje (\%) de investigadores en biotecnología según género. Costa Rica (1998-2002).

Fig. 1. Percentage (\%) of researchers in biotechnology according to kind(genre). Costa Rica (1998-2002).

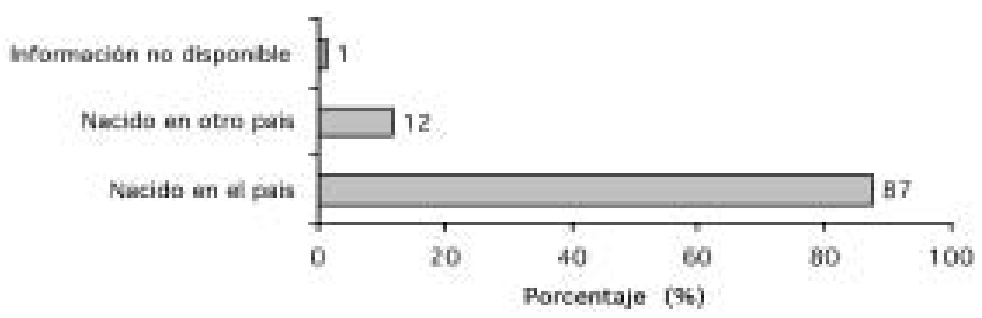

Fig. 2. Porcentaje (\%) de investigadores en biotecnología según nacionalidad. Costa Rica (1998-2002).

Fig. 2. Percentage (\%)of researchers in biotechnology according to nationality. Costa Rica (1998-2002). 


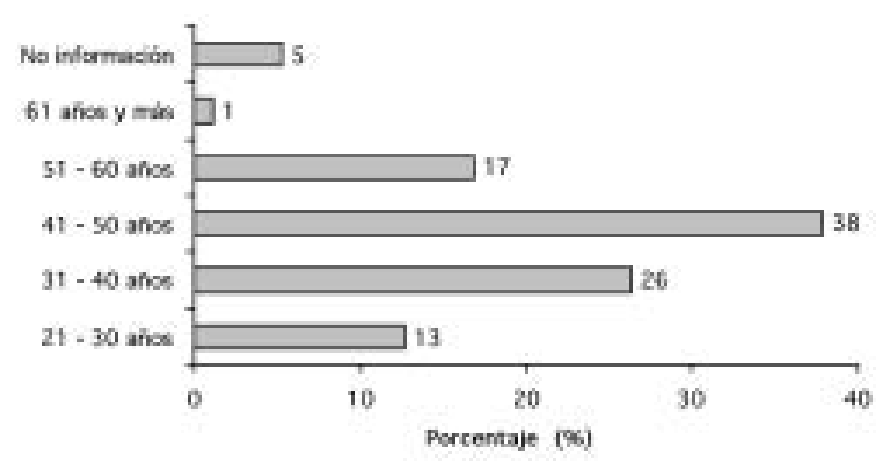

Fig. 3. Porcentaje (\%) de investigadores en biotecnología según edad. Costa Rica (1998-2002).

Fig. 3. Percentage (\%) of researchers in biotechnology according to age. Costa Rica (1998-2002).

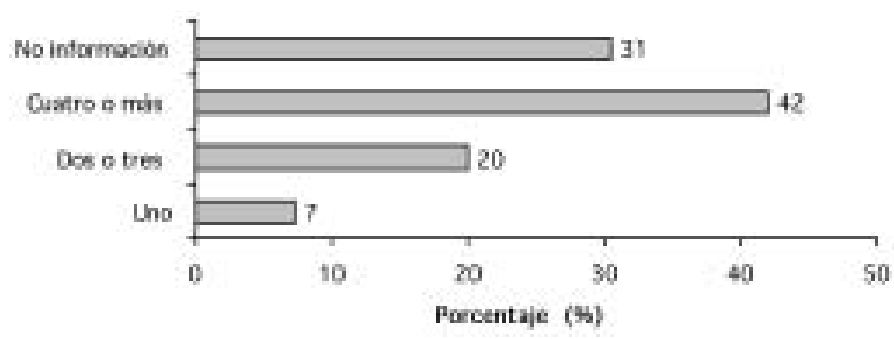

Fig. 4. Porcentaje (\%) de investigadores en biotecnología según número de proyectos. Costa Rica (1998-2002).

Fig. 4. Percentage (\%) of researchers in biotechnology according to number of projects. Costa Rica (1998-2002).

(Fig. 5). La gran mayoría (71\%) también realizan actividades docentes, son probablemente los que trabajan en las universidades estatales. Sólo un 29\% ofrece servicio de asesorías (Fig. 6). La figura 7 muestra que el área donde se ubican la mayor proporción $(57 \%)$ de investigadores es la de Bio-agro, seguida por la de Bio-salud (19\%) y la de Procesamiento de alimentos (9\%). Las otras áreas biotecnológicas a las que se dedican los investigadores se encuentran representadas en muy baja proporción: 5\% en Medio Ambiente, $4 \%$ en Productos Forestales, $2 \%$ en Bioprocesos y sólo un $1 \%$ en Acuicultura. El número de artículos científicos publicados por investigador en el periodo de estudio, es relativamente bajo, sólo un 7\% ha publicado más de 10 artículos, un $27 \%$ publicó de dos a cinco, un $20 \%$, de seis a diez, y un 39\% ningún artículo (Fig. 8).
Se obtuvo la información de 195 proyectos. Como en el caso de los investigadores, faltó la información de algunos proyectos del ITCR, del CATIE y de la UNA. En el sector privado, las empresas no proporcionaron información acerca de sus proyectos, ni de sus fuentes de financiamiento por motivos de confidencialidad. Por la misma razón, la mayor parte de centros de investigación pública no facilitaron los datos referentes a fuentes de financiamiento ni a su monto. Los 195 proyectos de biotecnología en Costa Rica, registrados en esta investigación, representarían aproximadamente un 10 a un $15 \%$ del total de proyectos de Ciencia y Tecnología desarrollados en las instituciones públicas del país, en el periodo de estudio (M. Valdez, datos sin publicar).

Con relación a las especialidades de los proyectos, la mayoría se clasifican en las 


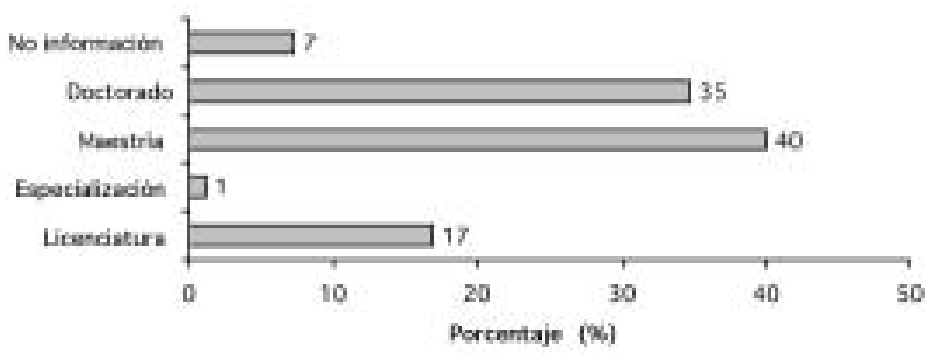

Fig. 5. Porcentaje (\%) de investigadores en biotecnología según último grado académico. Costa Rica (1998-2002).

Fig. 5. Percentage (\%) of researchers in biotechnology according to last academic degree. Costa Rica (1998-2002).

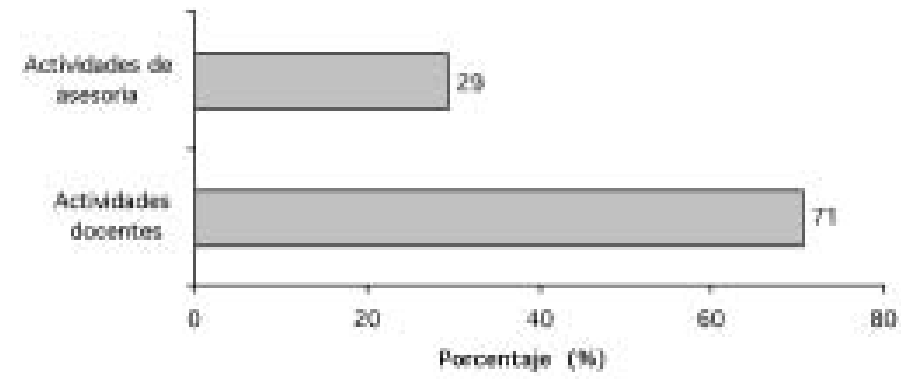

Fig. 6. Porcentaje (\%) de investigadores en biotecnología según actividad docente y de asesorías. Costa Rica (1998-2002).

Fig. 6. Percentage (\%) of researchers in biotechnology according to educational activity and of advisings. Costa Rica (1998-2002).

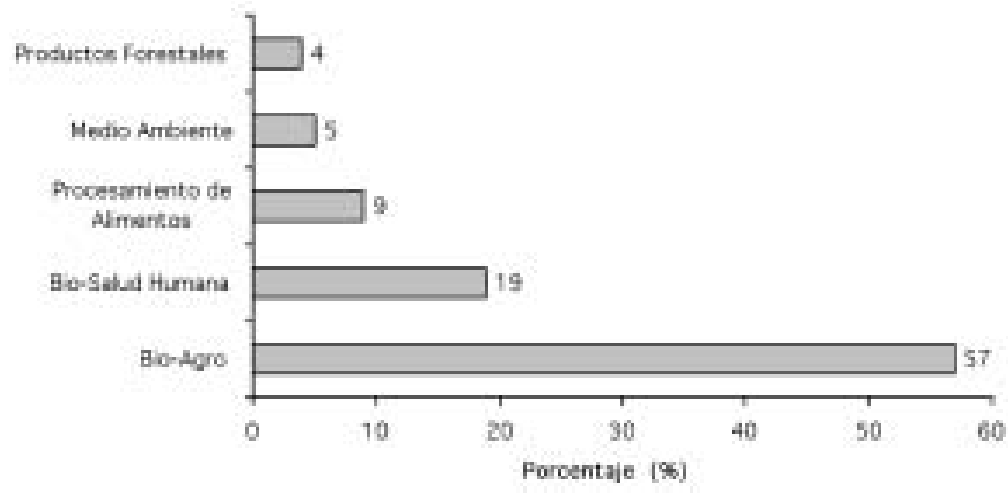

Fig.7. Porcentaje (\%) de investigadores según área biotecnológica. Costa Rica (1998-2002).

Fig.7. Percentage (\%) of researchers according to biotechnological area. Costa Rica (1998-2002). 


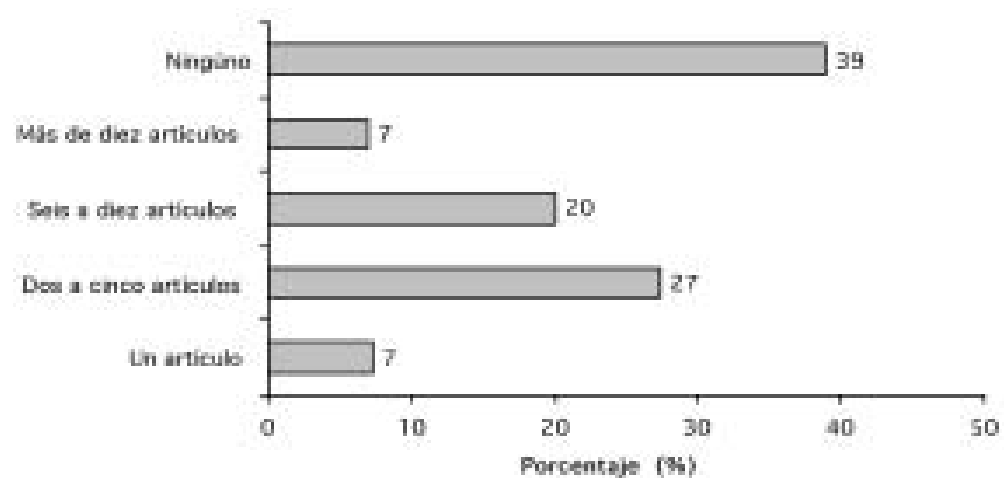

Fig. 8. Porcentaje (\%) de investigadores en biotecnología según número de artículos publicados. Costa Rica (1998-2002).

Fig. 8. Percentage (\%) of researchers in biotechnology according to number of published articles. Costa Rica (1998-2002).

categorías Bio-Agro (39\%) y Bio-Salud Humana (30\%) (Fig. 9). En orden de importancia le siguen: Procesamiento de Alimentos (7\%), Bio-informática (5\%), Productos Forestales (4\%), Bioprocesos (3\%), Medio Ambiente (2\%) y Acuicultura con sólo un $1 \%$. La figura 10 muestra que los sectores socio-económicos de aplicación tecnológica con el mayor número de proyectos son: Agro-Pecuario con un total de $37 \%$, que incluye un $30 \%$ para el área vegetal y un $7 \%$ para el área animal, y el de Salud Humana con un $35 \%$ de proyectos. En el sector industrial (alimentario y otros) sólo se ubican un $13 \%$ de proyectos.

Por otro lado, la mayoría de proyectos (58\%) tiene una duración menor a 3 años. Un 38\% entre 3 y 5 años, y sólo un $4 \%$ tienen una vigencia de 6 a 10 años. En cuanto al número de investigadores que participaron en cada proyecto, se obtuvieron los siguientes resultados: $24 \%$ de los proyectos con un sólo investigador; $54 \%$ con dos o tres; $20 \%$ con cuatro a seis, y un $2 \%$ con más de 7 investigadores.

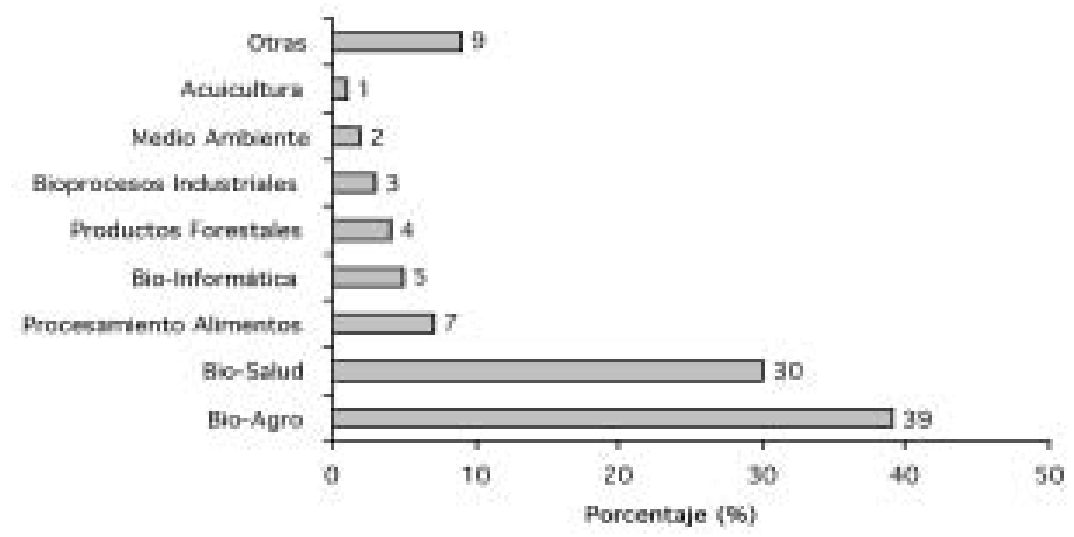

Fig. 9. Porcentaje (\%) de proyectos según área de la biotecnología. Costa Rica (1998-2002).

Fig. 9. Percentage (\%) of projects according to area of biotechnology. Costa Rica (1998-2002). 


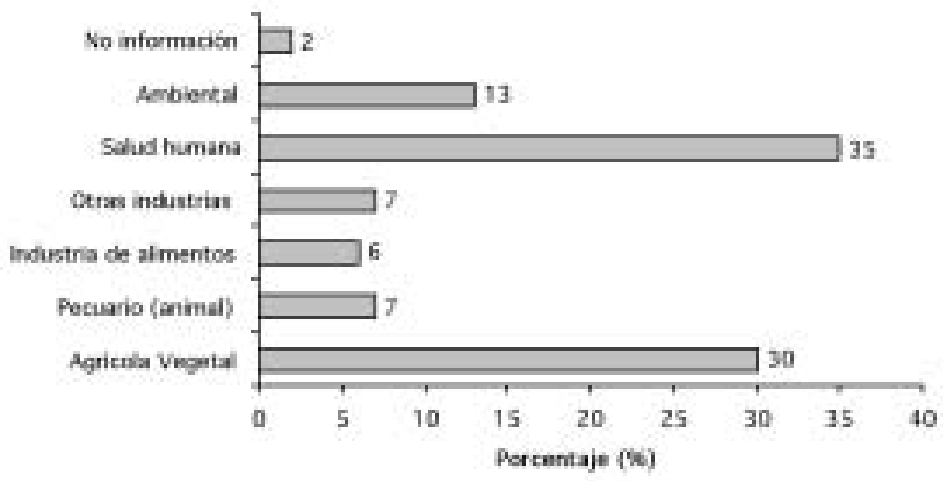

Fig. 10. Porcentaje (\%) de proyectos según sector de aplicación. Costa Rica (1998-2002).

Fig. 10. Percentage (\%) of projects according to sector of application. Costa Rica (1998-2002).

\section{DISCUSIÓN}

En los resultados de investigadores, sobresale la alta participación de las mujeres en la investigación biotecnológica del país, contrario a lo que ocurre en muchos otros países en desarrollo, donde su representación es aún muy baja (Sasson 1991). Esto puede deberse al acceso de las mujeres a niveles superiores de educación en Costa Rica, tal y como se muestra en los resultados de grado académico de los investigadores, donde un $75 \%$ tienen grado de Maestría y Doctorado. También, se observa una baja participación de investigadores extranjeros, no obstante, la alta cantidad de inmigrantes que recibe el país. Otro resultado interesante es el relacionado con la edad de los investigadores principales, donde se observa una baja participación de recursos humanos jóvenes, menores de 30 años. Éstos, usualmente realizan sus tesis de grado en esas edades, por lo que no fueron considerados en el estudio. Por otro lado, un $38 \%$ de los investigadores principales tiene entre 41 y 50 años, y un $18 \%$ es mayor de 51 años. Ello podría significar una limitación futura para la renovación de cuadros técnicos y científicos si no se realizan las evaluaciones necesarias, en la actualidad, de manera a planificar adecuadamente al respecto.

A pesar de que la mayoría de investigadores tienen cuatro o más proyectos bajo su responsabilidad, y que en los proyectos participan varios investigadores, el número de artículos científicos publicados por investigador, sea en revistas nacionales o internacionales, es bastante bajo. De la misma manera, el porcentaje que se dedica a brindar asesorías es relativamente modesto (29\%). Esto podría revelar un exceso de tareas docentes o de otra naturaleza. En efecto, un $71 \%$ de los investigadores encuestados se dedica a la docencia. Convendría entonces, realizar un estudio adicional, para elucidar las causas de la baja productividad científica de los investigadores en este campo.

Durante el estudio se tuvo algunas limitaciones en la recolección de la información, y en la clasificación de áreas y especialidades de los proyectos. Con la clasificación utilizada (Ver van Beuzekon 2001), se tuvo dudas en la identificación de la especialidad de algunos proyectos, por la ausencia de varias categorías en los formularios. Por lo anterior, en estudios futuros, se recomienda incluir las siguientes especialidades: i) Productos Naturales y Metabolitos Secundarios; ii) Medio ambiente: bioprospección; iii) Medio ambiente: conversión de desechos y subproductos agrícolas e industriales; iv) Bio-salud: biotecnología para sueros antiofídicos; v) Acuicultura: cultivos de algas marinas, de moluscos y de crustáceos. En la clasificación adoptada, la especialidad "acuicultura" incluye solamente cultivos de 
peces y extracción de productos de estos organismos. Esto podría explicar el bajo número de proyectos e investigadores encontrados en esta especialidad. Se conoce la existencia de al menos dos instituciones dedicadas a la acuicultura en el país (UCR y UNA), que posiblemente por esa razón, no fueron bien representados en el estudio.

Igualmente se recomienda utilizar palabras clave de cada una de las áreas, para la búsqueda inicial de proyectos en biotecnología que se encuentran registrados en los centros, ya que este método fue el que proporcionó una mejor información, como la obtenida para la UCR. Otra limitación de gran importancia de esta investigación, para continuar con este tipo de estudios en el futuro, es la imposibilidad de actualizar la información en la base de datos actual, debido a su conformación. Por ello, se sugiere reelaborar su diseño estructural y lógico, y trasladar la información ya recolectada, a una base de datos de mayor capacidad y flexibilidad, que permita mayor eficiencia, y que pueda ser consultada con más facilidad. Si esta base se llegara a colocar en un sitio de la internet, se podría mantener la información actualizada de manera permanente, por los mismos investigadores y centros de investigación (C. Hernández, com. personal).

De manera general, los resultados obtenidos muestran que se ha desarrollado una alta capacidad en investigación biotecnológica en el país, especialmente en el área de la biotecnología agrícola y de la asociada con la salud humana. No obstante, preocupa observar una baja proporción de proyectos en áreas relacionados con la actividad industrial (sólo un 13\%) y con el ambiente (bioremediación, bioprospección, etc.), en un país de alta biodiversidad como Costa Rica, que tiene una política tendiente al uso sostenible y a la conservación de sus recursos naturales.

La agricultura ha sido uno de los sectores de mayor importancia en la economía del país, aunque ha experimentado cambios negativos debido a la globalización y a la fluctuación de los precios de los principales productos agrícolas de exportación. En ese sentido, Costa Rica ha tomado decisiones estratégicas al incentivar el desarrollo de la biotecnología agrícola mediante el financiamiento de proyectos de investigación pública y privada por parte del Ministerio de Ciencia y Tecnología (MICIT) y del Consejo Nacional de Investigaciones Científicas y Tecnológicas (CONICIT).

Existen unos 17 centros de investigación pública que aplican técnicas biotecnológicas en el campo de la agricultura, que van desde la micropropagación in vitro, el diagnóstico de fitopatógenos, los métodos de control biológico de plagas, hasta las tecnologías de punta tales como la selección asistida por marcadores moleculares o por ingeniería genética, como en el caso de la UCR. Además de sus actividades de investigación, la mayoría de esos centros también ofrecen sus servicios a los productores o agricultores. A nivel privado, Arjona (2004), ha identificado unas 23 empresas que utilizan algún tipo de instrumento biotecnológico. Ellas se dedican a la producción y venta de plantas ornamentales, que son generadas por técnicas de cultivo in vitro, lo que permite su producción libre de patógenos. Así, este sector, contribuye de manera importante a la actividad productiva nacional a través de la exportación de sus productos. En los últimos 10 años, surgieron unas 4 empresas dedicadas al incremento de semilla "genética" transgénica, de cultivos como el algodón y la soya, para fines de exportación. Estas empresas "nodrizas" han contribuido a la construcción de la capacidad nacional en el manejo regulado de cultivos transgénicos, en conformidad con las normativas vigentes de bioseguridad ambiental establecidas por las autoridades fitosanitarias del Estado (MAG 2004). El desarrollo de la biotecnología agrícola por parte del sector privado del país, se puede ejemplificar con el mostrado por la empresa Agribiotecnología de Costa Rica, establecida en 1985. Ésta, fue la primera compañía dedicada a la micropropagación de plantas en América Central (Arias 1999). En los últimos años, esta empresa, además de sus actividades de micropropagación, ha realizado ensayos de campo para banano transgénico que expresa genes relacionados 
con el retardo de la maduración, en colaboración con la empresa transnacional "DNA Plant Technology". Otra compañía privada (UNIPO S. A.), también ha llevado a cabo recientemente, ensayos de campo para banano transgénico con tolerancia a la Sigatoka negra.

De manera semejante, en el sector salud, Costa Rica ha logrado avances muy importantes y significativos, como lo revelan los bajos índices de mortalidad infantil y de alta expectativa de vida de los habitantes (Estado de la Nación 2003). Como resultado de ese desarro1lo, se han generado un gran número de aplicaciones biotecnológicas en ese sector, que incluyen: estudios de genética humana, citogenética, diagnóstico molecular de enfermedades, diagnóstico prenatal de anormalidades genéticas, identificación de microorganismos patógenos, epidemiología del cáncer, producción de nuevos sueros antiofídicos con técnicas de ADN recombinante, etc. (CONARE 2003). Estos avances científicos han beneficiado a un gran porcentaje de la población costarricense, a través de los servicios que brinda el sistema nacional de salud del país, especialmente en lo que se refiere a los de diagnóstico y tratamiento de enfermedades genéticas como el cáncer.

En el sector forense y criminológico, también se ofrecen exámenes de huellas genéticas para elucidar homicidios o realizar pruebas de paternidad. Estos intrumentos biotecnológicos fueron transferidos desde los centros de investigación pública hasta los centros estatales del Poder Judicial (Arjona 2004). De la misma manera, la producción de sueros antiofídicos por parte del Instituto Clodomiro Picado de la UCR, contribuye a un tratamiento adecuado del accidente ofídico en Costa Rica y en otros países en América Latina. La aplicación de la biotecnología en empresas dedicadas a los sectores pecuario, acuícola o ambiental, es muy limitada. Se conoce sólo de una empresa (Avance Genético), dedicada al mejoramiento genético del ganado bovino con técnicas avanzadas de reproducción, tales como la inseminación artificial, y de una (ECODESOL), que brinda asesoramiento en el manejo de desechos y en el tratamiento de desechos biodegradables (Arjona 2004).

La presente investigación indica que el país tiene una buena infraestructura, un alto número de investigadores y de proyectos en el campo de la biotecnología, proporcionalmente al tamaño del país y de la población, acorde con lo señalado por Valdez y Sittenfeld (2002), y como se observa al comparar los indicadores costarricenses con los de otros países latinoamericanos, analizados por Testa (2003). El país, en las últimas décadas, ha dedicado cerca de un $10 \%$ del PIB a educación y salud, que lo ubica en buen lugar en el mundo, en cuanto a índice de desarrollo humano, según el Estado de la Nación (2003).

No obstante, el país parece tener varias debilidades o limitaciones para el desarrollo de la biotecnología, como son: pocas investigaciones en ciertas áreas que limitan su aplicación a sectores importantes de la actividad económica e industrial, bajo número de patentes o de aplicaciones industriales, o la poca participación del sector industrial en las actividades de investigación del sector público. Solleiro y Castañón (2002) señalan que los países latinoamericanos están llegando tardíamente a los mercados internacionales con sus productos y servicios biotecnológicos. Esto se encontraría muy relacionado con la estructura industrial de esos países, tradicionalmente renuentes a introducir cambios e innovaciones tecnológicas, y con poca inversión para actividades de investigación y desarrollo (I y D). Desde ese punto de vista, Costa Rica no sería la excepción, se invierte solamente un $0.35 \%$ del PIB en I y D en el país. No obstante, el número de investigadores y de publicaciones científicas es uno de los más altos de Latinoamérica, proporcionalmente al tamaño de su población. En los países desarrollados se invierte, por el contrario, de 2 a $3 \%$ del PIB para I y D en el sector público. A diferencia de los países desarrollados, el sector bancario nacional no es consciente del enorme potencial económico y social de las actividades biotecnológicas. De allí que el sector privado, industrial y empresarial, tiene pocas oportunidades para iniciar nuevas empresas de 
alta biotecnología en el país (Valdez y Sittenfeld 2002).

De manera general, puede considerarse que Costa Rica cuenta con una buena base de investigación científica y tecnológica, proporcionalmente al tamaño y grado de desarrollo socioeconómico del país. La comunidad científica nacional tiene además, una vinculación muy fuerte con sectores académicos, organizaciones y agencias internacionales científicas. Ello puede potenciar el desarrollo de una nueva industria biotecnológica, que fortalezca la economía y el desarrollo del país, que genere nuevos productos biotecnológicos (innovación tecnológica), o que dé valor agregado a los ya existentes.

\section{AGRADECIMIENTOS}

Los autores agradecen a Gabriela Solano, Jeannette Acón y Ana Lucía Calderón por la asistencia en la obtención de información. A Elmer Guillermo García por la revisión del manuscrito. Este trabajo fue financiado por la Universidad de Costa Rica, a través del Centro Nacional de Ciencia y Tecnología de Alimentos y la Comisión Institucional de Biotecnología de la Vicerrectoría de Investigación, y por la Organización de Estados Americanos (OEA), a través del proyecto SIMBIOSIS.

\section{RESUMEN}

Se realizó un estudio del estado de la biotecnología en Costa Rica como parte del proyecto "SIMBIOSIS: Programa Cooperativo para la Construcción de Indicadores en Biotecnología adaptados a los países de América Latina y el Caribe, para motivar la aplicación y transferencia de tecnologías industriales". El estudio se enfocó en dos ítemes: "investigadores" y "proyectos de investigación", desarrollados en Costa Rica entre 1998 y 2002. Se construyó una base de datos a partir de la cual se obtuvieron indicadores para los investigadores, relacionados con aspectos como género, edad, desempeño como docentes, número de proyectos, funciones, grado académico, área de especialidad y número de publicaciones. Los indicadores determinados para los proyectos de investigación se vinculan con los temas de: especialidades, sector socio-ecónomico de aplicación, duración y número de investigadores por proyecto.
Entre las principales conclusiones obtenidas a nivel nacional cabe mencionar la alta participación de las mujeres en esta área de investigación (54\%); la baja participación de recursos humanos jóvenes como investigadores (13\% menores de 30 años), y que la mayoría de los investigadores, con altos grados académicos, tienen 4 o más proyectos a su cargo $(42 \%)$. Con relación a las especialidades de los proyectos, la mayoría se clasifican en la categoría BioAgro (39\%) mientras que en Acuicultura sólo se encontró un $1 \%$ del total. Los sectores de aplicación con el mayor número de proyectos son: Agropecuario (37\%) y Salud Humana (35\%). Se discuten las principales fortalezas y limitaciones para el desarrollo de la biotecnología en Costa Rica, para contribuir a una mejor definición de políticas de desarrollo científico y tecnológico del país.

\section{REFERENCIAS}

Arias, O. 1999. Addressing market needs through biotechnology: the example of Agribiotecnología de Costa Rica. In J. Komen, C. Falconi \& H. Hernández (eds.). Agricultural Biotechnology Policy Seminar. pp. 147-150. The Hague/Mexico, D. F., Intermediary Biotechnology Service/CamBioTec.

Arjona, R. 2004. Desarrollo de un Marco Nacional en Bioseguridad para Costa Rica. In A. May (ed.). Proyecto UNEP-GEF. Consultado en octubre 2004, http://www.protecnet.go.cr/bioseguridad

Estado de la Nación. 2003. Estado de la Nación en Desarrollo Humano Sostenible. Consultado en julio 2004, http://www.estadonacion.or.cr/

MAG (Ministerio de Agricultura y Ganadería). 2004. Gerencia Técnica de Biotecnología. Consultado en octubre de 2004, http://www.mag.go.cr/servicios/fito sanitario.html

CONARE (Consejo Nacional de Rectores). 2003. Programa BID II. Biotecnología, justificación de sub-áreas. Costa Rica, Febrero de 2003. 22 p.

Sasson, A. 1985. Les biotechnologies: defis et promesses. U.N.E.S.C.O, Paris, France. 336 p.

Sasson, A. 1988. Biotechnologies and development. UNESCO, Technical Centre for Agricultural and Rural Cooperation (CTA), Paris, France. 361 p.

Sasson, A. 1991. Biotechnologies in perspective: Socioeconomic implications for developing countries. UNESCO, Presses Universitaires de France. 166 p.

Serageldin, I. 1999. Biotechnology and food security in the $21^{\text {st }}$ century. Science $285: 387-389$.

Solleiro, J. L. \& R. Castañón. 2002. La Biotecnología en América Latina: panorama al año 2002. pp: 115-132. 
In J. Verástegui (ed.). CamBioTec, Iniciativa Canadiense-Latinoamericana en Biotecnología para el Desarrollo Sustentable. IDRC, Canadá.

Testa, P. 2003. Indicadores de las capacidades de investigación en biotecnología: análisis comparativo de Colombia, Costa Rica, México y Venezuela. Programa Cooperativo para la Costrucción de Indicadores en Biotecnología y Tecnología de Alimentos: Taller de Indicadores en Biotecnología, Bogotá, Colombia, Noviembre de 2003.

UCR (Universidad de Costa Rica). 2001. Programa Institucional de Biotecnología: 2001-2004. Comisión Institucional de Biotecnología, Vicerrectoría de Investigación, San José, Costa Rica. 21 p.
Valdez, M. \& A. Sittenfeld. 2002. Current State of Modern Agricultural Biotechnology in Costa Rica. OECD Global Forum on Knowledge Economy - Biotechnology. Paris, France, 18-19 Noviembre 2002.

Ver van Beuzekom, B. 2001. Biotechnology Statistics in OECD Member Countries: Compendium of Existing National Statistics. Organisation for Economic Cooperation and Development, Directorate for Science, Technology and Industry STI Working Papers.

Zeledón, R. 1988. Significado de la nueva biotecnología para América Latina y el Caribe. La nueva biotecnología en agricultura y salud. Instituto Interamericano de Cooperación para la Agricultura, (IICA), Serie 7 de Documentos de Programas, San José, Costa Rica. 